\title{
Adapting to the Unpredictable. My Experience as a Neurosurgeon-Hypnologist Offering Treatment to Health Professionals in a Covid-19 Converted Hospital
}

\section{Cividini A* \\ Department of Neurosurgery, ASST Ovest Milanese \\ Legnano Hospital, Italy \\ *Corresponding author: Cividini A, Department of Neurosurgery, ASST Ovest Milanese Legnano Hospital, via Papa Giovanni Paolo II, 20025 Legnano (MI), Italy}

Received: April 12, 2021; Accepted: April 16, 2021;

Published: April 23, 2021

\section{Letter to Editor}

At the beginning of 2020, Covid-19 pandemic has deeply and quickly changed healthcare transforming our hospitals and challenging health professionals both as clinicians and humans. We found ourselves fighting an invisible, unknown enemy, facing an unpredictable disease and had no choice but adapting and reacting to an overgrowing emergency situation. Whole hospitals were dedicated to Covid-19 patients and many specialized physicians had to quickly learn about treating Sars-Cov-2 infection. Over the last year better knowledge of pathophysiology and treatment as well as the establishment of redesigned organizational models have contributed to reduce the role of chance, fear and unexpected, but as the pandemic keeps unfolding, healthcare workers are still under pressure. As a result, they are exposed to a high risk of post-traumatic stress disorder as well as burn-out syndrome. As a neurosurgeon working in a Covid-converted hospital and an hypnologist I realized it was my duty to use my skills to help colleagues in need. I started offering hypnosis sessions to help colleagues to stay in-balance and react to this situation in a resilient way. In this letter I underline some features which I find to be peculiar to the Covid-19 pandemic (some of them more relevant to the first waves), describe their consequences on health professionals' life and suggest the potential role of hypnosis as a tool to promote mental health.

- Loss of anchors: entire hospitals or wards have been turned into Covid-dedicated areas, thus requiring professionals to change their daily habits, to quickly learn new skills and to be exposed to a constantly changing situation. The sudden and continuous loss of such anchors is deeply destabilizing.

- Change in social engagement: self-quarantine, limiting of social activities, avoidance of close contacts and having to wear disposable equipment partially covering the face impact on the usual way of being socially involved. Paradoxically, family and community can't offer a safe shelter in a moment of major need.

- Identification: before vaccines became available, the high number of health professionals affected by the disease, the unfortunately common unavailability of proper personal protective equipment and the need for a constant monitoring of self health status, often lead to identification with patients or victims.

- $\quad$ Lack of control: such a level of uncertainty and lack of knowledge is new to many young doctors who have been raised studying diagnosis and treatment of mostly well-defined and known conditions.

- $\quad$ Social media impact: being connected inevitably exposes users to a constant flow of bad or fake news. Besides being a reason to recall a healthier digital lifestyle, this leads to rumination and prevents people from using spare time to fully recover from the fatigue.

Carers who asked for hypnosis sessions mainly complained about sleep-related problems, panic attacks, rumination, anxiety and fear (of dying alone, of not being able to make plans for the future, of not being able to reconnect with loved ones, of transmitting the disease to family members or colleagues, of not being able to breathe). The reiteration of such symptoms puts health professionals on the dangerous road to burn-out and eventually PTSD. Hypnosis, as well as all disciplines aimed at improving mental health (mind-body techniques, mindfulness, meditation, yoga, deep relaxation..) may offer help in preventing them.

Healthcarers who required hypnosis sessions were invited to join after or before their shift. I treated clinicians and nurses, aged 25-55 yo, mainly female. A short introductory talk was aimed at identifying their perception of the situation and potential topics to work on during the session. Being all the patients highly motivated in feeling better, almost none had difficulties in trance-induction. Sessions had a mean duration of $45-60^{\prime}$ and were held in the hospital facilities. I found the following techniques being particularly useful:

- Focus on breathing: being Covid-19 disease mainly a respiratory illness and due to the need to work wearing uncomfortable masks, focus on breathing is crucial. Reconnecting people to their natural breath may help in keeping a healthy connection to the body and preventing panic attacks.

- Safe place: visualizing and then anchoring a "safe place" help subjects to realize that they can actively choose to feel better going back to their safe place every time they need it.

- Metaphors: the use of subject-tailored metaphors is particularly effective, giving immediate relief as well as working on a longer-lasting deeper level.

- Temporal dissociation and retrieving happy memories: in difficult and uncertain times, reestablishing a connection with happy memories and with past achievements helps subjects to discover their own value, be self-confident and get rid of harmful helplessness feeling. 
- Future reorienting: for all the aforementioned reasons future reorienting techniques may be useful for those people feeling afraid of not being able to look beyond this challenging times

All subjects were satisfied with the session, found it helpful and most of them asked to repeat the treatment.

I hope this letter may contribute to increase institutions' awareness on the need for measures aimed at maintaining and supporting mental health of all professionals involved in fighting the ongoing pandemic. Besides, it's also addressed to professionals as a reminder to promptly identify stress-related red flags and ask for help. Hypnosis is a powerful, effective and safe tool. 